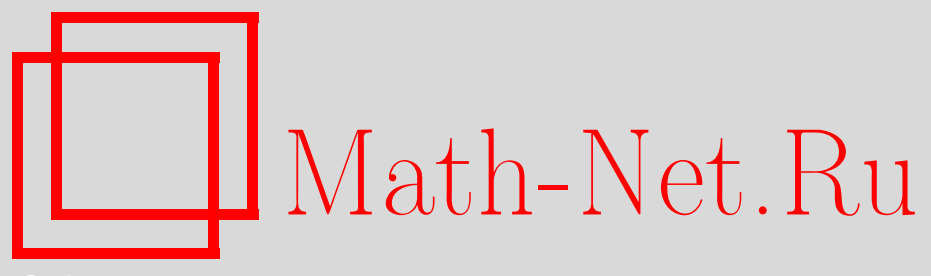

В. З. Гринес, Е. Я. Гуревич, В. С. Медведев, О. В. Починка, О включении в поток диффеоморфизмов МорсаСмейла на многообразиях размерности, большей двух, Матем. заметки, 2012, том 91, выпуск 5, 791-794

DOI: https://doi.org/10.4213/mzm9365

Использование Общероссийского математического портала Math-Net.Ru подразумевает, что вы прочитали и согласны с пользовательским соглашением http://www . mathnet.ru/rus/agreement

Параметры загрузки:

IP : 18.234 .156 .22

26 апреля 2023 г., 18:26:27

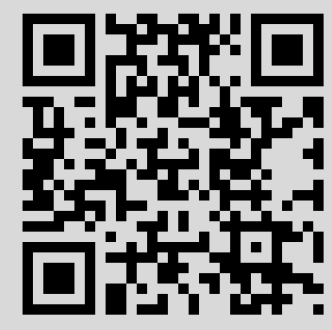




\section{О включении в поток диффеоморфизмов Морса-Смейла на многообразиях размерности, большей двух}

\section{В. З. Гринес, Е. Я. Гуревич, В. С. Медведев, О. В. Починка}

Пусть $M^{n}$ - гладкое связное замкнутое многообразие $M^{n}$ размерности $n, \operatorname{Diff}^{r}\left(M^{n}\right)-$ пространство всех диффеоморфизмов на $M^{n}$ гладкости $C^{r}, r \geqslant 1$. Напомним, что диффеоморфизм $f \in \operatorname{Diff}^{1}\left(M^{n}\right)$ называется диффеоморфизмом Морса-Смейла, если выполняются следующие условия:

- неблуждающее множество $\Omega(f)$ конечно и состоит из гиперболических периодических точек;

- устойчивые и неустойчивые многообразия периодических точек пересекаются трансверсально.

Будем говорить, что диффеоморфизм $f: M^{n} \rightarrow M^{n}$ включается в топологический (гладкий) поток, если существует топологический (гладкий) поток $X^{t}$ на $M^{n}$ такой, что $f$ является сдвигом на единицу времени вдоль траекторий потока $X^{t}$. Из работ [1], [2], в которых доказана структурная устойчивость диффеоморфизмов Морса-Смейла, следует, что для любого многообразия $M^{n}$ существует открытое в $\operatorname{Diff}^{1}\left(M^{n}\right)$ множество диффеоморфизмов Морса-Смейла, включающихся в топологический поток. Палис доказал в [1] следующие необходимые условия включения диффеоморфизма $f$ Морса-Смейла в топологический поток:

1) множество $\Omega(f)$ совпадает с множеством Fix $(f)$ неподвижных точек;

2) ограничение диффеоморфизма $f$ на каждое инвариантное многообразие любой неподвижной точки $p \in \Omega(f)$ сохраняет его ориентацию;

3) если для различных седловых точек $p, q \in \Omega(f)$ пересечение $W^{s}(p) \cap W^{u}(q)$ непусто, то каждая его компонента связности не является замкнутым множеством.

Более того, он показал, что при $n=2$ эти условия являются достаточными. В этой же работе была поставлена проблема нахождения соответствующих условий в случае большей размерности.

Следует отметить, что проблема в гладкой ситуации значительно отличается от топологической. Так в работе [3] доказано, что множество $C^{2}$-диффеоморфизмов, включающихся в $C^{1}$-гладкий поток, нигде не плотно в пространстве диффеоморфизмов Морса-Смейла.

В настоящей заметке получены необходимые и достаточные условия включения в топологический поток сохраняющих ориентацию диффеоморфизмов Морса-Смейла, объединенных в класс $G_{k}\left(M^{n}\right)(n>2)$ следующими условиями, выполняющимися для любого $f \in G_{k}\left(M^{n}\right):$

1) $\Omega(f)=\operatorname{Fix}(f)$;

2) множество седловых точек состоит в точности из $k>0$ седловых неподвижных точек ${ }^{1}$, причем устойчивое многообразие $W^{s}(\sigma)$ любой седловой неподвижной точки $\sigma \in \Omega(f)$ имеет размерность $n-1$;

Работа выполнена при поддержке правительства Российской Федерации (грант 11.G34.31.0039), Российского фонда фундаментальных исследований (гранты №o 11-01-12056-офи-м-2011, 12-0100672-а) и гранта Минобрнауки РФ в рамках государственного задания на оказание услуг в 2012-2014 гг. подведомственными высшими учебными заведениями (шифр заявки 1.1907.2011).

${ }^{1}$ Если $k=0$, то $\Omega(f)$ состоит в точности из одного стока и одного источника, все диффеоморфизмы с таким неблуждающим множеством вкладываются в топологический поток. 
3) для любых двух различных седловых точек $p, q \in \Omega(f)$ пересечение $W^{s}(p) \cap W^{u}(q)$ пусто.

Из условий, выделяющих класс $G_{k}\left(M^{n}\right)$, следует, что неблуждающее множество любого диффеоморфизма $f \in G_{k}\left(M^{n}\right)$ содержит в точности один источник и $k+1$ стоковую точку, а несущее многообразие $M^{n}$ диффеоморфно $n$-сфере. Отметим, что любой диффеоморфизм $f \in G_{k}\left(M^{n}\right)$ удовлетворяет всем необходимым условиям включения в поток, сформулированным выше.

Теорема 1. Если $n \geqslant 4$, то любой диффеоморфизм $f \in G_{k}\left(M^{n}\right)$ включается в топологический поток.

Теорема 1 является следствием результатов, полученных в работах [4], [5].

В случае $n=3$, как следует из работ [6], [7], уже в классе $G_{1}\left(M^{3}\right)$ существуют диффеоморфизмы, не включающиеся в поток. Этот эффект связан с возможностью дикого вложения замыкания сепаратрис седловых неподвижных точек в несущее многообразие. Для пояснения этого эффекта приведем точные определения ручного и дикого вложения.

Напомним, что устойчивой (неустойчивой) сепаратрисой седловой периодической точки $\sigma$ диффеоморфизма $f$ называется компонента связности многообразия $W^{s}(\sigma) \backslash \sigma$ $\left(W^{u}(\sigma) \backslash \sigma\right)$. Пусть $\alpha$ - источниковая неподвижная точка диффеоморфизма $f \in G_{k}\left(M^{3}\right)$ Морса-Смейла. Из гиперболичности $\alpha$ следует, что $W^{u}(\alpha)$ гомеоморфно пространству $\mathbb{R}^{3}$. Из определения класса $G_{k}\left(M^{3}\right)$ следует, что все сепаратрисы седловых точек диффеоморфизма $f$, принадлежащие $W^{u}(\alpha)$, двумерны. Будем обозначать их объединение через $\mathscr{L}_{\alpha}$. Положим $F_{\alpha}=\mathscr{L}_{\alpha} \cup \alpha$ и назовем $F_{\alpha}$ двумерным пучком сепаратрис. Аналогично определяется пучок одномерных сепаратрис $F_{\omega}$, принадлежащий устойчивому многообразию стоковой точки $\omega$.

Пусть $\mathbb{S}^{2}=\left\{(x, y, z) \in \mathbb{R}^{3} \mid x^{2}+y^{2}+z^{2}=1\right\},\left\{s_{1}, \ldots, s_{\mu}\right\}\left(\left\{p_{1}, \ldots, p_{\nu}\right\}\right)-$ попарно непересекающиеся простые замкнутые кривые (попарно несовпадающие точки), принадлежащие сфере $\mathbb{S}^{2}$, и $C_{j}$ - конус в $\mathbb{R}^{3}$ с вершиной в начале координат и направляющей $s_{j}$, $j \in\{1, \ldots, \mu\}$ ( $L_{i}-$ луч, выходящий из начала координат и проходящий через точку $p_{i}$, $i \in\{1, \ldots, \nu\})$. Совокупность $\mathbb{F}^{2}=\bigcup_{j=1}^{\mu} C_{j}\left(\mathbb{F}^{1}=\bigcup_{j=1}^{\nu} L_{j}\right)$ будем называть стандартным двумерным (одномерным) пучком.

Подмножество $F^{i} \subset \mathbb{R}^{3}$, снабженное индуцированной топологией и гомеоморфное $\mathbb{F}^{i}$, $i \in\{1,2\}$, будем называть одномерным или двумерным пучком. При этом пучок $F^{i}$ будем называть ручным, если существует гомеоморфизм $H: \mathbb{R}^{3} \rightarrow \mathbb{R}^{3}$ такой, что $H\left(F^{i}\right)=\mathbb{F}^{i}$;

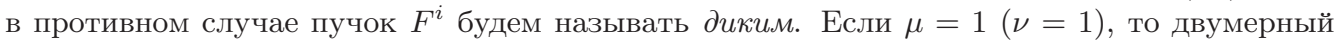
(одномерный) пучок вырождается в конус (дугу). Первые примеры диких конусов и диких дуг были приведены Дж. Александером в 1924 году, Е. Артином и Р. Фоксом в 1948 году. Отметим, что ручность каждого из элементов, входящих в пучок $F^{i} \subset \mathbb{R}^{3}$, еще не является гарантией того, что пучок в целом будет ручным. Например, в работе [8] построен пример так называемого умеренно дикого одномерного пучка, т.е. такого дикого пучка, что любой содержащийся в нем пучок из $\nu^{\prime}<\nu$ дуг является ручным.

Определение 1. Двумерный пучок сепаратрис $F_{\alpha}$ назовем ручным, если существует гомеоморфизм $H_{\alpha}: W^{u}(\alpha) \rightarrow \mathbb{R}^{3}$, отображающий $F_{\alpha}$ на стандартный двумерный пучок. В противном случае будем говорить, что пучок сепаратрис $F_{\alpha}$ является диким. Если ручной (дикий) пучок $F_{\alpha}$ содержит только одну сепаратрису, то будем называть эту сепаратрису ручной (дикой).

Аналогично определяется одномерный ручной (дикий) пучок сепаратрис $F_{\omega}$.

В работах [6], [7] построены примеры диффеоморфизмов из класса $G_{1}\left(M^{3}\right)$, имеющих дико вложенные одномерную и двумерную сепаратрису седла, в работе [9] построены диффеоморфизмы из класса $G_{k}\left(S^{3}\right), k \geqslant 2$, одномерные сепаратрисы которых образуют умеренно дикие пучки. Как будет следовать из леммы 1 , такие диффеоморфизмы не включаются ни в какие потоки. Сюрпризом оказался тот факт, что условие ручности всех 
пучков сепаратрис седловых неподвижных точек диффеоморфизма $f \in G_{k}\left(M^{3}\right)$ так же не является достаточным для вложения диффеморфизма $f$ в поток, а именно, справедливо следующее предложение.

ПредлОЖениЕ 1. Существует не включающийся в поток диффеоморфизм $f \in G_{4}\left(S^{3}\right)$, все пучки сепаратрис которого являются ручными.

Как оказалось, необходимое условие включения диффеоморфизма $f \in G_{k}\left(M^{3}\right)$ в поток заключается в более сильном, нежели ручность, требовании.

Пусть $A: \mathbb{R}^{3} \rightarrow \mathbb{R}^{3}$ - линейное растяжение евклидова пространства $\mathbb{R}^{3}$, определяемое формулой $A\left(x_{1}, x_{2}, x_{3}\right)=\left(2 x_{1}, 2 x_{2}, 2 x_{3}\right)$.

ОПределение 2 . Пучок сепаратрис $F_{\alpha}$ называется тривиально вложенным, если существует гомеоморфизм $h_{\alpha}: W^{u}(\alpha) \rightarrow \mathbb{R}^{3}$, отображающий пучок $F_{\alpha}$ на стандартный двумерный пучок, и удовлетворяющий условию $\left.f\right|_{W^{u}(\alpha)}=\left.h_{\alpha}^{-1} A h_{\alpha}\right|_{W^{u}(\alpha)}$.

Аналогично определяется тривиально вложенный одномерный пучок сепаратрис $F_{\omega}$.

ПредлОЖениЕ 2. Существует не включающийся в поток диффеоморфизм $f \in G_{5}\left(S^{3}\right)$ такой, что для любой стоковой точки $\omega \in \Omega(f)$ пучок $F_{\omega}$ является тривиальным.

Лемма 1. Пусть $f \in G_{k}\left(M^{3}\right)$. Если $f$ включается в топологический поток, то все пучки сепаратрис диффеоморфизма $f$ тривиальны.

Основной результат работы заключается в следующей теореме.

Теорема 2. Диффеоморфизм $f \in G_{k}\left(M^{3}\right)$ вкладывается в топологический поток тогда и только тогда, когда пучок $F_{\alpha}$ тривиально вложен.

Необходимость условия теоремы 2 следует из леммы 1. Доказательство достаточности сводится к получению такого полного топологического инварианта в классе диффеоморфизмов из $G_{k}\left(M^{3}\right)$ с тривиальными двумерными пучками, который можно реализовать диффеоморфизмом, являющимся сдвигом на единицу времени вдоль траекторий потока. Таким инвариантом является граф $\Gamma(f)$ диффеоморфизма $f$, множество вершин которого изоморфно множеству неподвижных точек, а множество ребер - множеству сепаратрис седловых неподвижных точек.

Теорема 3. Пусть $f, f^{\prime} \in G_{k}\left(M^{3}\right)$ и пучки $F_{\alpha}, F_{\alpha^{\prime}}$ тривиальны. Диффеоморфизмы $f$, $f^{\prime}$ топологически сопряжены тогда и только тогда, когда графы $\Gamma(f), \Gamma\left(f^{\prime}\right)$ изоморфны.

Из работы [10] следует, что для потоков Морса-Смейла, удовлетворяющих условиям, аналогичным условиям, определяющим класс диффеоморфизмов $G_{k}\left(M^{n}\right)$, граф является полным топологическим инвариантом, и любой абстрактный допустимый граф (обладающий теми же свойствами, что и графы потоков из рассматриваемого класса) реализуется таким потоком. Отсюда следует, что для любого диффеоморфизма $f \in G_{k}\left(M^{n}\right)$, удовлетворяющего теореме 3 , существует поток $X^{t}$, граф которого изоморфен графу $\Gamma(f)$. В силу леммы 1 все пучки сепаратрис диффеоморфизма $X^{1}$, полученного сдвигом на единицу времени вдоль потока $X^{t}$ являются тривиальными. Тогда диффеоморфизм $f$ топологически сопряжен с $X^{1}$, т.е. существует гомеоморфизм $h: M^{n} \rightarrow M^{n}$ такой, что $f=h X^{1} h^{-1}$. Следовательно, диффеоморфизм $f$ вкладывается в топологический поток $Y^{t}=h X^{t} h^{-1}$.

\section{СПИСОК ЦИТИРОВАННОЙ ЛИТЕРАТУРЫ}

[1] J. Palis, Topology, 8:4 (1969), 385-404. [2] Дж. Палис, С. Смайл, Математика. Сб. пер., 13:2 (1969), 145-155. [3] М.И. Брин, Изв. вузов. Матем., 1972, № 8, 19-25. [4] В. З. Гринес, Е. Я. Гуревич, В. С. Медведев, Дифференциальные уравнения и динамические системы, Сборник статей, Тр. МИАН, 261, МАИК, М., 2008, 61-86. [5] В. З. Гринес, 
Е.Я. Гуревич, В.С. Медведев, Дифференииальные уравнения и динамические системы, Сборник статей, Тр. МИАН, 270, МАИК, М., 2010, 62-85. [6] D. Pixton, Topology, 16:2 (1977), 167-172. [7] C. Bonatti, V. Grines, J. Dynam. Control Systems, 6:4 (2000), 579-602. [8] H. Debrunner, R. Fox, Duke Math. J., 27:3 (1960), 425-429. [9] O. Pochinka, Univ. Iagel. Acta Math., 47 (2009), 149-154. [10] С. Ю. Пилюгин, Дифферени. уравнения, 14:2 (1978), $245-254$.

\section{В. З. Гринес}

Нижегородская государственная сельскохозяйственная академия E-mail: vgrines@yandex.ru

\section{Е. Я. Гуревич}

Нижегородский государственный университет им. Н. И. Лобачевского E-mail: elena_gurevich@list.ru

\section{В. С. Медведев}

Нижегородский государственный университет им. Н. И. Лобачевского E-mail: medvedev@unn.ac.ru

\section{О. В. Починка}

Нижегородский государственный университет им. Н. И. Лобачевского E-mail: olga-pochinka@yandex.ru 